\title{
Reporte de caso: adenocarcinoma pancreático ductal asociado a hipertensión portal izquierda
}

Case report: Pancreatic ductal adenocarcinoma associated to portal hypertension

Adrian Pérez-González ${ }^{1 *}$, Martha Sánchez-Silva ${ }^{2}$, Ma Guadalupe Gómez-Pérez $^{3}$, Oscar Quiroz-Castro ${ }^{1}$, Violeta Navarro-Martínez ${ }^{1}$ y Paloma P. Pérez-Ladrón de Guevara ${ }^{1}$

${ }^{1}$ Servicio de Imagenología; ${ }^{2}$ Alta Especialidad en Resonancia Magnética Corporal; ${ }^{3}$ Departamento de Resonancia Magnética. Hospital Ángeles Pedregal, Ciudad de México, México

\section{RESUMEN}

La hipertensión portal izquierda es una entidad clínica rara y poco descrita que muchas veces es subdiagnosticada porque se confunde con otra patología, como la hipertensión portal usual. Estas dos patologías varían mucho en cuanto a etiología, presentación clínica y tratamiento, por lo que es indispensable conocer la existencia de ambas para poder discernir entre una y otra y tomar la mejor decisión terapéutica. En cuanto al enfoque de radiología, es necesario conocer esta entidad, su etiología frecuente, asociaciones y complicaciones, para saber qué hallazgos hay que buscar intencionadamente en los estudios cuando se enfrente un sangrado del tubo digestivo no por varices esofágicas sino por varices gástricas o por sangrado de ambas. Se presenta un caso recién diagnosticado para aprovecharlo y realizar una revisión breve de la literatura que existe en cuanto a frecuencia, fisiopatología, cuadro clínico y hallazgos de la hipertensión portal izquierda.

Palabras clave: Hipertensión portal izquierda. Varices gástricas. Melena. Adenocarcinoma páncreas. 


\section{ABSTRACT}

Left portal hypertension it's a rare uncommon clinical entity baerly described that's many times underdiagnosed since it is confused with another pathology such as usual portal hypertension. This two pathologies vary a lot regarding aetiology, clinical presentation and treatment therefore it is essential to know the existence of both etiologies to discern in between them and take the best therapeutic decisión. As for the radiologic approach it is needed to know this entity, is frequent aetiology, associations and implications in order to know which findings too look for intentionally in the studies whenever we are studying a patient for digestive tract bleeding not for esophageal varices but for gastric varices or both. In the present paper is presented a case of recently diagnosed left portal hypertension to make a brief revision of the literature regarding frequency, physiopathology and clinical findings.

Key words: Left portal hypertension. Gastric varices. Blood in stool. Pancreatic adenocarcinoma.

\section{INTRODUCCIÓN}

En el siglo XVII, se descubrieron cambios estructurales en la circulación portal que causan sangrado gastrointestinal, y no fue hasta 1902 que Gilbert y Carnot introdujeron el término hipertensión portal para describir esta condición. Se define como el incremento patológico de la presión hidrostática intravascular en el sistema portal ${ }^{1,2}$.

La hipertensión portal se asocia frecuentemente a cirrosis hepática, sin embargo, no es la única causa. En México, la cirrosis hepática fue la cuarta causa de mortalidad en 2010 en personas de entre 25 y 45 años $^{3}$.

La presión portal normal es de 5 a $10 \mathrm{mmHg}$, medida en la vena porta y en sus colaterales. Cuando la presión se eleva fuera de este rango ya se considera patológico, y tiene expresión clínica a partir de los $12 \mathrm{mmHg}$.
En 1939, Greenwald, et al. describieron la fisiopatología de otro síndrome llamado síndrome de hipertensión portal izquierda, como causa importante de sangrado del tubo digestivo alto por varices gástricas secundario a patología pancreática ${ }^{4}$.

Este síndrome de hipertensión portal izquierda es causado por la obstrucción, en la mayoría de los casos, de la vena esplénica.

Se presenta clásicamente por una tríada que consiste en varices gástricas aisladas, esplenomegalia y función hepática normal ${ }^{5}$.

Las varices gástricas aisladas resultan de la trombosis u obstrucción de la vena esplénica, con cambios en la presión del vaso y flujo retrógrado.

La causa más frecuente es la patología pancreática, que incluye la pancreatitis y neoplasias pancreáticas ${ }^{6}$. 


\section{PRESENTACIÓN DE CASO}

Paciente masculino de 59 años de edad, originario de la Ciudad de México, con estudios a nivel de licenciatura, ateo.

Refiere antecedentes familiares de padre finado por complicaciones de una fractura de cadera, sin enfermedades crónico-degenerativas de importancia. Madre finada por complicaciones de enfermedad pulmonar obstructiva crónica. Tiene tres hijos, aparentemente sanos.

Habita un departamento propio con su esposa, niega convivencia con animales domésticos. Cambio de ropa y baño diario, aseo dental una vez por día. Etilismo social, nivel de actividad sedentario y niega toxicomanías.

De los antecedentes personales patológicos de importancia, refiere antecedente de transfusión de ocho paquetes globulares en 2017 por sangrado del tubo digestivo con hemoglobina de $3.4 \mathrm{mg} / \mathrm{dl}$. En cuanto a los antecedentes quirúrgicos, se le realizó hernioplastia umbilical hace 4 años, hemicolectomía por diverticulitis y apendicetomía en 2016.

Padece diabetes mellitus tipo 2, diagnosticada en 2015, en tratamiento con insulina glargina, canagliflozina y metformina. Hiperplasia prostática benigna diagnosticada en 2017, en tratamiento con doxazosina y dutasterida. Gota diagnosticada en 2000, en tratamiento con alopurinol. Hipertrigliceridemia diagnosticada en el mismo año y tratada con fenofibrato.

Inicia su padecimiento actual el 12 de marzo del 2018 con evacuaciones melénicas en una ocasión, al momento de su ingreso en urgencias niega dolor abdominal, náuseas, vómitos $\mathrm{u}$ otros síntomas asociados. Únicamente refiere hiporexia. A la exploración física se encuentra con signos vitales estables, con palidez de tegumentos +++.

Se decide transfundir un paquete globular en urgencias $\mathrm{y}$, posteriormente, se realizan estudios de laboratorio y gabinete de rutina, y se encuentran los siguientes hallazgos relevantes: Hemoglobina $7.5 \mathrm{mg} / \mathrm{dl}$. Se solicita endoscopia de urgencia que reporta coágulos de varices gástricas con hemorragia activa intensa, por lo que se ligó y se aplicó escleroterapia. Durante el procedimiento se transfundieron otros dos paquetes globulares.

Los resultados de laboratorio a su ingreso al servicio de urgencias se muestra en la Tabla 1.

Se realizó un estudio de angiotomografía axial computarizada, cuyo hallazgo principal

TaBla 1. Resultados de laboratorio al ingreso en el servicio de urgencias

\begin{tabular}{|l|c|}
\hline Tiempo de protrombina & $18.2 \mathrm{~s}$ \\
\hline Tiempo parcial de tromboplastina & $23.5 \mathrm{~s}$ \\
\hline Razón normalizada internacional & 1.31 \\
\hline Hemoglobina & $7.5 \mathrm{~g} / \mathrm{dl}$ \\
\hline Plaquetas & 136,000 plaquetas/mcl \\
\hline Leucocitos & 7.8 \\
\hline Bilirrubinas totales & $0.37 \mathrm{mg} / \mathrm{dl}$ \\
\hline Bilirrubina directa & $0.16 \mathrm{mg} / \mathrm{dl}$ \\
\hline Bilirrubina indirecta & $0.21 \mathrm{mg} / \mathrm{dl}$ \\
\hline Gama glutamil transferasa & $14.3 \mathrm{UI} / \mathrm{l}$ \\
\hline DHL & $88 \mathrm{UI} / \mathrm{l}$ \\
\hline Lípidos totales & $474 \mathrm{mg} / \mathrm{dl}$ \\
\hline Amilasa & $24 \mathrm{Ul} / \mathrm{dl}$ \\
\hline Lipasa & $18 \mathrm{Ul} / \mathrm{dl}$ \\
\hline
\end{tabular}



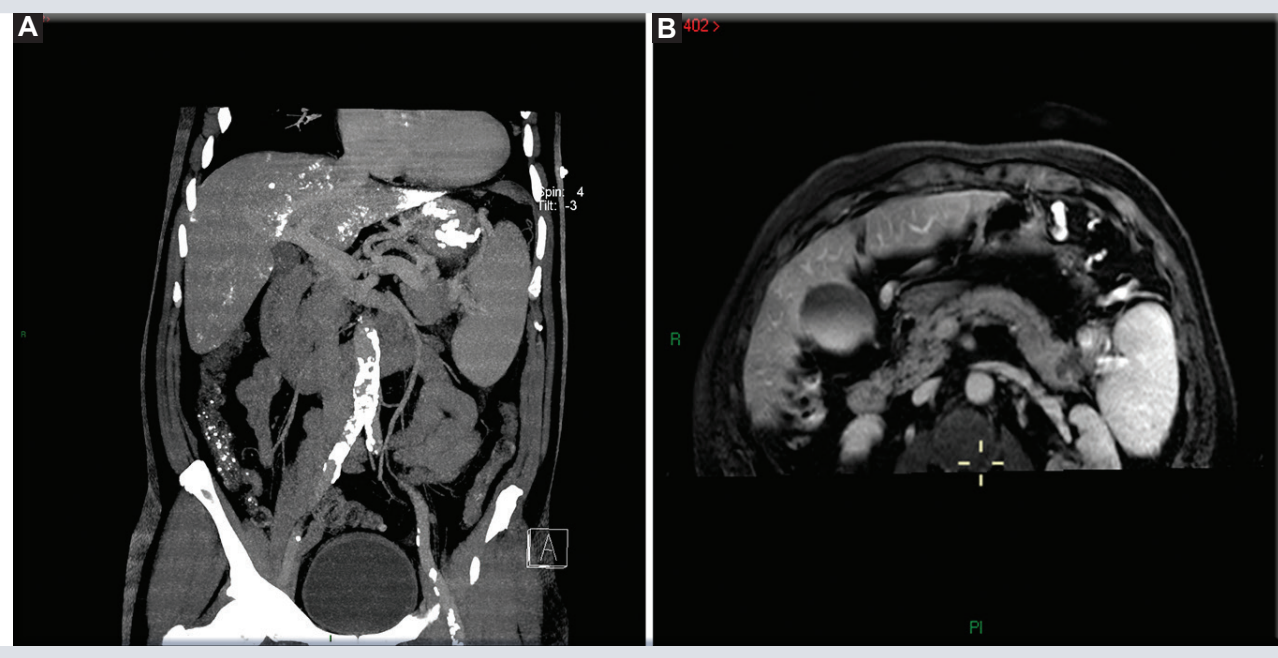

Figura 1. A: angiotomografía de abdomen en la que se visualiza probable infiltración a la vena esplénica y esplenomegalia. B: estudio de resonancia magnética en el que se muestra infiltración de la lesión pancreática a la vena esplénica.

fue una masa en la cola del páncreas con bordes espiculados, con aparente infiltración a la vena esplénica y esplenomegalia (Fig. 1).

Basándose en los hallazgos previamente descritos, se realizó pancreatectomía distal y esplenectomía, y se enviaron las piezas quirúrgicas al servicio de patología para su análisis, con los resultados siguientes: adenocarcinoma pancreático ductal con grados variables de diferenciación (moderadamente a poco diferenciado) de la cola del páncreas, con diferenciación mucinosa focal de $3 \mathrm{~cm}$ de longitud mayor unifocal. Extensión al tejido adiposo peripancreático posterior. Invasión perivascular venosa a la vena esplénica y perineural multifocal. Esplenomegalia congestiva $(494 \mathrm{~g} / 17 \mathrm{~cm})$

\section{DISCUSIÓN}

La hipertensión portal izquierda, también se conoce como hipertensión esplenoportal, hipertensión portal segmental, regional, localizada o compartamental. Su fisiopatología fue descrita por primera vez en 1939 por Greenwald, et al. ${ }^{4,7}$.

Se considera una entidad rara, presente en menos del 5\% de todos los pacientes con hipertensión portal. De hecho, hay quien sugiere que el nombre de hipertensión portal izquierda está mal empleado, ya que la presión portal usualmente se encuentra en rangos normales en estos pacientes ${ }^{7,8}$.

Los parámetros de funcionamiento hepático normalmente se encuentran conservados, y como hallazgo frecuente hay varices gástricas, en lugar de las clásicas varices esofágicas encontradas en la hipertensión portal usual ${ }^{9,10}$.

La vena porta es el vaso principal del sistema venoso portal, drena sangre del tracto gastrointestinal y del bazo al hígado. Mide aproximadamente $8 \mathrm{~cm}$ de largo en los adultos, con un diámetro máximo de $13 \mathrm{~mm}$ (Fig. 2). 


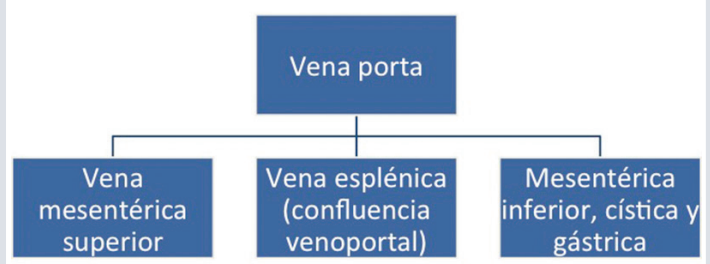

Figura 2. Formación de la vena porta.

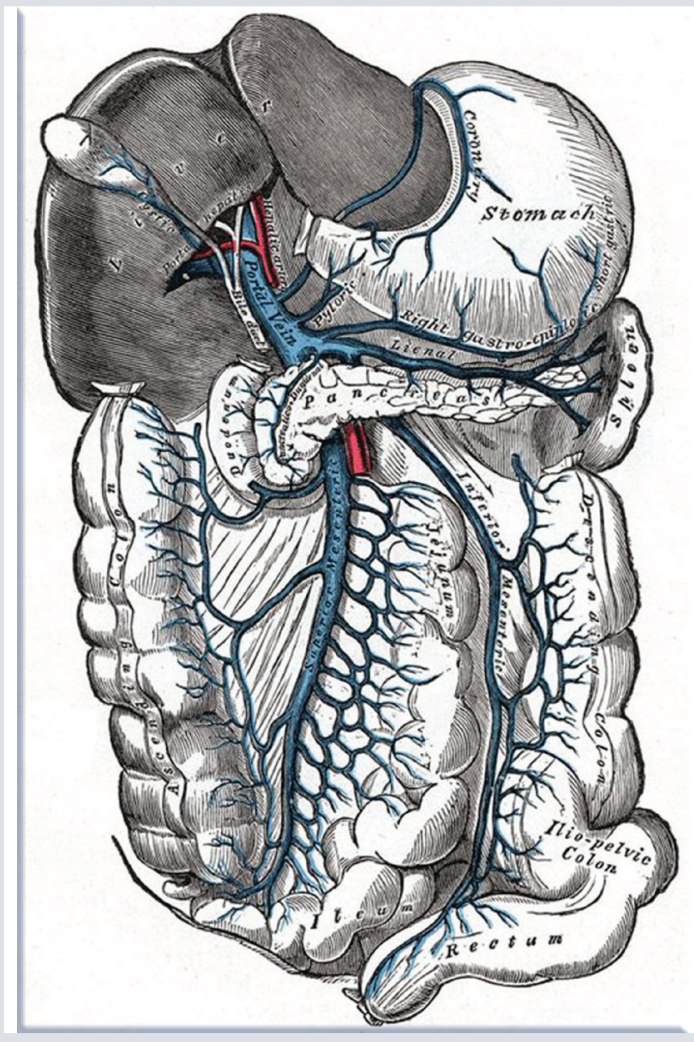

Figura 3. Esquema de la vena porta.

Cortesía del Dr. Jeremy Jones, Radiopaedia.org, rID: 36242.

Se origina por detrás del cuello del páncreas, clásicamente se forma por la confluencia de la vena esplénica, vena mesentérica superior e inferior ${ }^{11}$ (Fig. 3).

A la vez, la vena esplénica, que confluye a la porta, se forma por vasos tributarios que emergen del hilio esplénico en el ligamento esplenorrenal. Corre a través de este ligamento en asociación cercana a la arteria esplénica. Justo por detrás del cuello del páncreas confluye con la vena mesentérica superior hacia la porta (Fig. 4).

Las venas tributarias de la vena esplénica son: la vena gástrica corta, la vena gastroepiploica izquierda, la vena mesentérica inferior y venas pancreáticas. $Y$ de estas tributarias es que se deriva la sintomatología inicial (varices gástricas) de esta entidad ${ }^{12,13}$.

Dentro de las causas de este síndrome se encuentran las que se describen en la Tabla $2^{5,14}$.

\section{Fisiopatología}

Como se ha comentado, la vena esplénica es susceptible a lesiones secundarias a patología del páncreas por su proximidad. La oclusión de la vena esplénica resulta en presión retrógrada que se transmite a sus anastomosis con las venas gástrica corta, gastroepiploicas y, subsecuentemente, a través de la vena coronaria al sistema portal. Esto resulta en flujo reverso y formación de varices gástricas.

La hipertensión se confina al lado izquierdo del sistema porta y, es por eso, que su presentación clínica es muy diferente a la hipertensión portal generalizada.

Las varices gástricas aisladas ocurren en la hipertensión portal izquierda, y las varices gastroesofágicas generalizadas en la hipertensión portal generalizada, sin embargo, aun así, se debe excluir también de inicio la 


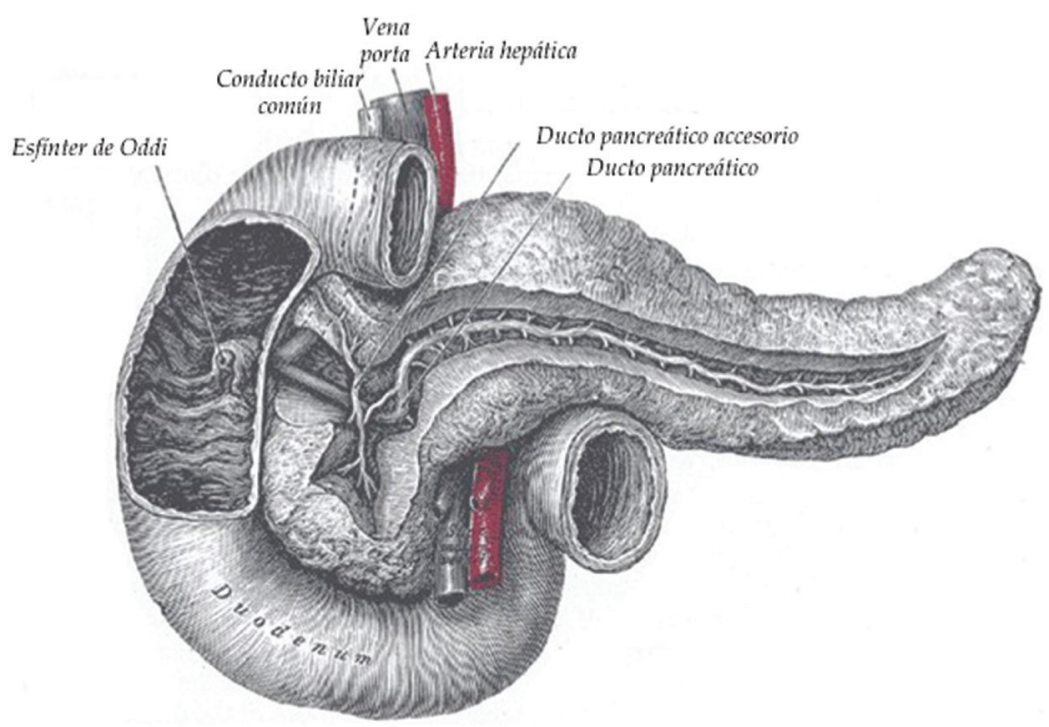

Figura 4. Relación del páncreas y el duodeno con la esplénica.

Cortesía del Dr. Jeremy Jones, Radiopaedia.org, rID: 36231.

TABLA 2. Causas de hipertensión portal izquierda ${ }^{5,14}$

\begin{tabular}{|c|c|c|}
\hline Causa & Mecanismo & $\begin{array}{l}\text { MANIFESTACIÓN } \\
\text { DEL SANGRAdO }\end{array}$ \\
\hline \multicolumn{3}{|c|}{ Lesiones pancreáticas benignas } \\
\hline Pancreatitis aguda & $\begin{array}{l}\text { Trombosis } \\
\text { esplénica }\end{array}$ & $\begin{array}{l}\text { Varices gástricas } \\
\text { aisladas }\end{array}$ \\
\hline Pseudoquiste pancreático & $\begin{array}{l}\text { Trombosis } \\
\text { esplénica }\end{array}$ & $\begin{array}{l}\text { Varices gástricas } \\
\text { aisladas }\end{array}$ \\
\hline Pseudotumor pancreático & $\begin{array}{l}\text { Compresión } \\
\text { esplénica }\end{array}$ & $\begin{array}{l}\text { Varices gastroe- } \\
\text { sofágicas }\end{array}$ \\
\hline \multicolumn{3}{|l|}{ Cáncer de páncreas } \\
\hline $\begin{array}{l}\text { Adenocarcinoma } \\
\text { pancreático }\end{array}$ & $\begin{array}{l}\text { Trombo o } \\
\text { compresión }\end{array}$ & $\begin{array}{l}\text { Varices gástricas } \\
\text { aisladas }\end{array}$ \\
\hline \multicolumn{3}{|l|}{ Lesión vascular } \\
\hline $\begin{array}{l}\text { Trombosis esplénica } \\
\text { aislada }\end{array}$ & Trombo & $\begin{array}{l}\text { Varices gástricas } \\
\text { aisladas }\end{array}$ \\
\hline $\begin{array}{l}\text { Pseudoaneurisma de } \\
\text { arteria esplénica }\end{array}$ & $\begin{array}{l}\text { Compresión } \\
\text { y trombosis }\end{array}$ & $\begin{array}{l}\text { Varices gástricas } \\
\text { aisladas }\end{array}$ \\
\hline \multicolumn{3}{|l|}{ Otras } \\
\hline Linfadenitis tuberculosa & Compresión & $\begin{array}{l}\text { Varices gástricas } \\
\text { aisladas }\end{array}$ \\
\hline
\end{tabular}

hipertensión portal generalizada en las primeras $^{6,15}$.

\section{Tratamiento}

El manejo de esta condición, tradicionalmente, incluye la resección quirúrgica, a ser posible, de la causa subyacente, combinada con esplenectomía ${ }^{15,16}$.

La esplenectomía decrementa el flujo de sangre al sistema porta por la ligadura de la arteria esplénica, lo que resulta en descompresión de las varices gástricas. Sin embargo, la esplenectomía se reserva para casos de sangrado intenso de las varices gástricas o casos graves de hipertensión portal izquierda $^{17,18}$.

El pronóstico de los pacientes depende de la etiología primaria de la hipertensión ${ }^{19}$.

\section{CONCLUSIÓN}

En pacientes con sangrado del tubo digestivo alto asociado a várices gástricas, debe 
considerarse la posibilidad de hipertensión portal izquierda, entidad poco frecuente. En el caso de nuestro paciente, la causa primaria fue un adenocarcinoma ductal pancreático con infiltración a la vena esplénica. Los métodos de imagen, como la tomografía y la resonancia magnética, ayudan a delimitar la lesión en el páncreas, detectar invasión vascular, valorar otros órganos y descartar otras etiologías. El médico radiólogo debe conocer la asociación de neoplasias pancreáticas y el desarrollo de hipertensión portal izquierda para poder caracterizar las lesiones.

\section{CONFLICTO DE INTERESES}

Los autores declaran no tener conflicto de interés en relación a este artículo.

\section{FINANCIAMIENTO}

El financiamiento del presente trabajo corre por cuenta de los autores.

\section{RESPONSABILIDADES ÉTICAS}

Protección de personas y animales. Los autores declaran que para esta investigación no se han realizado experimentos en seres humanos ni en animales.

Confidencialidad de los datos. Los autores declaran que en este artículo no aparecen datos de pacientes.

\section{Derecho a la privacidad y consentimiento informado. Los autores declaran que en}

este artículo no aparecen datos de pacientes.

\section{BIBLIOGRAFÍA}

1. Ibarrola-Calleja JL, Núñez F, Rodríguez M, Ordóñez-Céspedes J. Hipertensión portal. Acta Médica Grupo Ángeles. 2011;9(2):83-91.

2. Arun J, Vijay H. Hipertensión portal. Patobiología, evaluación y tratamiento. New Jersey: Humana Press; 2005.

3. Instituto Mexicano del Seguro Social. Diagnóstico y tratamiento de várices esofágicas en el adulto. Guía de Evidencias y Recomendaciones: Guía de Práctica Clínica. México: IMSS; 2017

4. Greenwald HM, Wasch MG. The roentgenologic demonstration of esophageal varices as a diagnostic aid in chronic thrombosis of the splenic vein. J Pediatr. 1939;14:57-65.

5. Joya MD, Del Valle P, Martínez M, Herrera N, Agud JL. Hipertensión portal izquierda con sangrado por varices gástricas como forma de presentación de un hipernefroma. An Med Inter (Madrid). 2005;21(6):283-4.

6. Thompson R, Taylor M, McKie LD, Diamond T. Sinistral portal hypertension. Ulster Med J. 2006;75(3):175-7.

7. Fernández A, Almeida N, Ferreira AM, Casela A, Gomes D, Portela F, et al. Left- Sided Portal Hypertension: A Sinister Entity. GE Port J Gastroenterol. 2015;22(6):234-9.

8. Koklu S, Yuksel O, Arhan M, Coban S, Başar O, Yolcu OF, et al. Report of 24 left-sided portal hypertension cases: a single-center prospective cohort study. Dig Dis Sci. 2005;50:976-82.

9. Koklu S, Coban S, Yuksel O, Arhan M. Left-sided portal hypertension. Dig Dis Sci. 2007;52:1141-9.

10. Wang L, Liu GJ, Chen YX, Dong HP, Wang LX. Sinistral portal hypertension: clinical features and surgical treatment of chronic splenic vein occlusion. Med Princ Pract. 2012;21:20-3.

11. Schmidt S, Demartines N, Soler L, Schnyder P, Denys A. Portal vein normal anatomy and variants: implication for liver surgery and portal vein embolization. Semin Intervent Radiol. 2008;25 (2):86-91.

12. Drake RL, Vogl AW, Mitchell AWM. Gray's Anatomy for Students. $2^{\text {nd }}$ ed. Churchill Livingstone; 2009

13. Last RJ, McMinn R. Last's anatomy, regional and applied. Edinburgh: Churchill Livingstone; 1994.

14. Seifeldin H, Bortman J, Orosey M, Cappell MS. Case report and systematic literature review of a novel etiology of sinistral portal hypertension presenting with UGI bleeding Left gastric artery pseudoaneurysm compressing the splenic vein treated by embolization of the pseudoaneurysm. Medicine. Baltimore. 2017;96(13):e6413.

15. Sakorafas GH, Sarr MG, Farley DR, Farnell MB. The significance of sinistral portal hypertension complicating chronic pancreatitis. Am J Surg. 2000;179(2):129-33.

16. Pereira P, Peixoto A. Left-Sided Portal Hypertension: A Clinical Challenge. GE Port J Gastroenterol. 2015;22(6):231-3.

17. Loftus JP, Nagorney DM, Illstrup D, Kunselman AR. Sinistral portal hypertension. Splenectomy or expectant management. Ann Surg. 1993;217(1):35-40.

18. Adams DB, Mauterer DJ, Vujic IJ, Anderson MC. Preoperative control of splenic artery in ow in patients with splenic venous occlusion. South Med J. 1990;83(9):1021-4.

19. Hwang TL, Jan YY, Jeng LB, Chen MF, Hung CF, Chiu CT. The different manifestation and outcome between pancreatitis and pancreatic malignancy with left-sided portal hypertension. Int Surg. 1999;84(3):209-12. 